University for Business and Technology in Kosovo

UBT Knowledge Center

UBT International Conference

2016 UBT International Conference

Oct 28th, 9:00 AM - Oct 30th, 5:00 PM

\title{
Radial Structure Graph Based Mathematical for Large Distribution Networks
}

Ines Bula

University for Business and Technology, ines.bula@ubt-uni.net

Valmir Hoxha

University for Business and Technology, valmir.hoxha@ubt-uni.net

Edin Bula

University for Business and Technology, edinbula2@gmail.com

Follow this and additional works at: https://knowledgecenter.ubt-uni.net/conference

Part of the Engineering Commons, and the Physical Sciences and Mathematics Commons

\section{Recommended Citation}

Bula, Ines; Hoxha, Valmir; and Bula, Edin, "Radial Structure Graph Based Mathematical for Large Distribution Networks" (2016). UBT International Conference. 42.

https://knowledgecenter.ubt-uni.net/conference/2016/all-events/42

This Event is brought to you for free and open access by the Publication and Journals at UBT Knowledge Center. It has been accepted for inclusion in UBT International Conference by an authorized administrator of UBT Knowledge Center. For more information, please contact knowledge.center@ubt-uni.net. 
RADIAL STRUCTURE GRAPH -BASED MATHEMATICAL MODEL FOR LARGE DISTRIBUTION NETWORKS

\title{
RADIAL STRUCTURE GRAPH -BASED MATHEMATICAL MODEL FOR LARGE DISTRIBUTION NETWORKS
}

\author{
Ines Bula ${ }^{1}$, Valmir Hoxha ${ }^{2}$, Edin Bula ${ }^{3}$ \\ ${ }^{1,2,3}$ UBT - Higher Education Institution, Lagjja Kalabria, 10000 p.n., \\ Prishtine, Kosovo \\ \{ines.bula ${ }^{1}$, valmir.hoxha $\left.{ }^{2}\right\}$ ubt-uni.net \\ edinbula2@gmail.com ${ }^{3}$
}

\begin{abstract}
In the modern electrical distribution network and utility companies a steady increase in the need for high quality and safe delivery of electricity to consumers is evident, which requires an adequate distribution network, that is a network with high quality voltage and low loss of energy, with a small number of failures and equipment failure, etc. In this paper is proposed a way of representing the mathematical model of distribution networks. For calculations of energy applications, the best way for representation distribution network is in form of graph of the radial structure. By presenting the distribution network in the form of a radial structure graph, which consists of nodes and branches, possible through the use of specialized compensation algorithms for the calculation of steady state and fault analysis. Advantages of these algorithms, as compared to other algorithms are much shorter time needed for calculation and much higher accuracy of calculation results.
\end{abstract}

Keywords: Distribution Network, Distribution Management System, Model Topology Service, Bus / Branch model, Mathematical Model of Distribution Networks.

\section{Introduction}

In the modern electrical distribution network and utility companies a steady increase in the need for high quality and safe delivery of electricity to consumers is evident, which requires an adequate distribution network, that is a network with high quality voltage and low loss of energy, with a small number of failures and equipment failure etc. [1-2]

That quality of electricity supply can be ensured by investing in expensive power equipment and by applying significantly less expensive automation equipment and sophisticated systems to automate the management of distribution networks. The most sophisticated advancement is Distribution Management System (DMS). As an industrial product, the DMS is still in development. [1-2]

This system provides a very high quality performance of all technical tasks in distribution companies: surveillance and control, analysis, management, operation and development planning of distribution networks, etc. DMS represents a software platform based on a set of applications and services necessary to ensure the effective functionality of distribution networks and consumer companies in order to achieve a secure electricity supply at minimum cost. [1-2] 


\section{Book of Proceedings}

International Conference on Mechatronics, Sciences in Energy Efficiency Engineering, System Engineering and Robotics

The system of energy applications, is based on a set very complex algorithms and mathematical procedures, and is certainly the most important component of DMS. The complexity of these procedures is a result of the very large size of the distribution network, a strong sense of uncertainty of data, a small number of data on the current situation and so on. The basis on which all energy applications rely presents a mathematical model of the distribution network (DN).

Mathematical model of the network processes the static data elements of the network and slow changing variable data (status of switching equipment) to determine the topological structure of the distribution network considered. Number of entities in the power system is large, resulting in the application of algorithms for topology modeling, affecting the speed of calculating energy applications. In the paper, a bus / Branch network model is used, which has no information about switchboard equipment, measurements and other details. This model was implemented by using object-oriented methodologies. The key features of this methodology are inheritance, aggregation, association and polymorphism that give better software implementation.

\section{ARCHITECTURE DMS}

DMS application, generally speaking, make the following system components (subsystems):

- Graphical User Interface (GUI) - customers who represent presentation level

- DMS server that represents the level of the application

- Historical data representing the level of the database.

DMS application server consists of a series of independent software services that interact together and give a response to the input that can be a customer requirements and changes in the state of devices in the field. Each of these services is independent with its internal representation of the physical system, providing an interface for interaction with other services. Services are divided into several functional groups, as shown in Figure 1. [3] 
RADIAL STRUCTURE GRAPH -BASED MATHEMATICAL MODEL FOR LARGE DISTRIBUTION NETWORKS

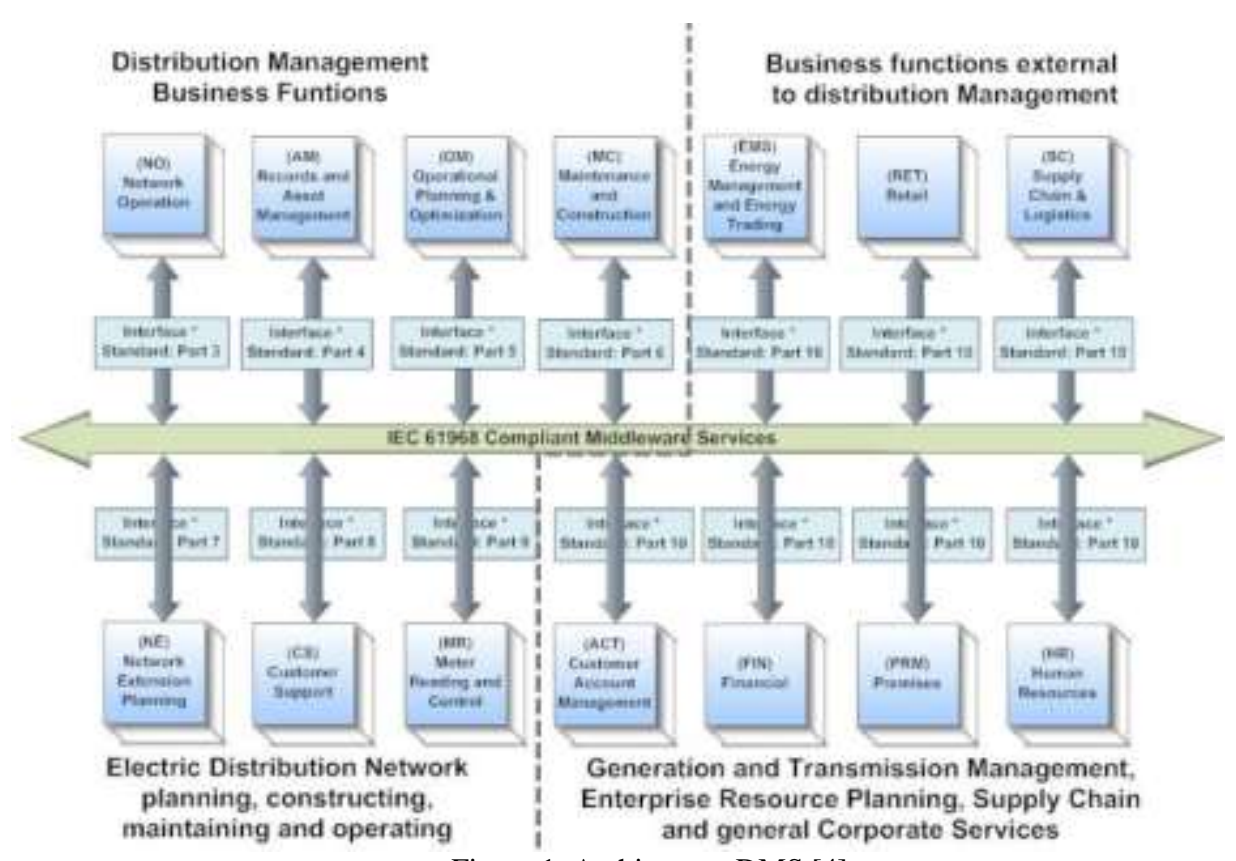

Figure 1. Architecture DMS [4]

All services together constitute a single context. The context is a set of data and services abovehim that are working over a state. It allows the operation of DMS in a safe and quick way. Context can be:

- Operational - when the connection to SCADA system (DMS Real Time)

- Simulation and

- Context for testing and data entry.[2-3]

\section{Model Topology Service}

Model Topology Service (MTS) is a service that is responsible for the creation of a mathematical model. It contains a representation of a power distribution system, which is based on the current state of the switching equipment. This service connect's together static and dynamic information about the elements. Data elements are placed in appropriate vectors. MTS when determining the topology it creates an internal model to be used in a variety of DMS calculations. [5]

\subsection{The internal model}

The model is the basis for all energy functions, because it contains all the network information presented in the form of a graph. Graf DN consists of:

- nodes,

- branches, 
Book of Proceedings

International Conference on Mechatronics, Sciences in Energy Efficiency Engineering,

System Engineering and Robotics

- shunts.

These elements of DN are interconnected across the field, and are collectively referred to as the ends of the field. Fields are complex elements that are essential in the formation of the graph, for connecting and contain all the other elements DN: switching equipment, measuring and protective devices and so on. The fields and ends fields are elements of a graph. Facilities of the power system in the internal model are presented with classes and relationships that exist between these classes. Only basic classes that are used to create an internal model are shown in Figure 2.

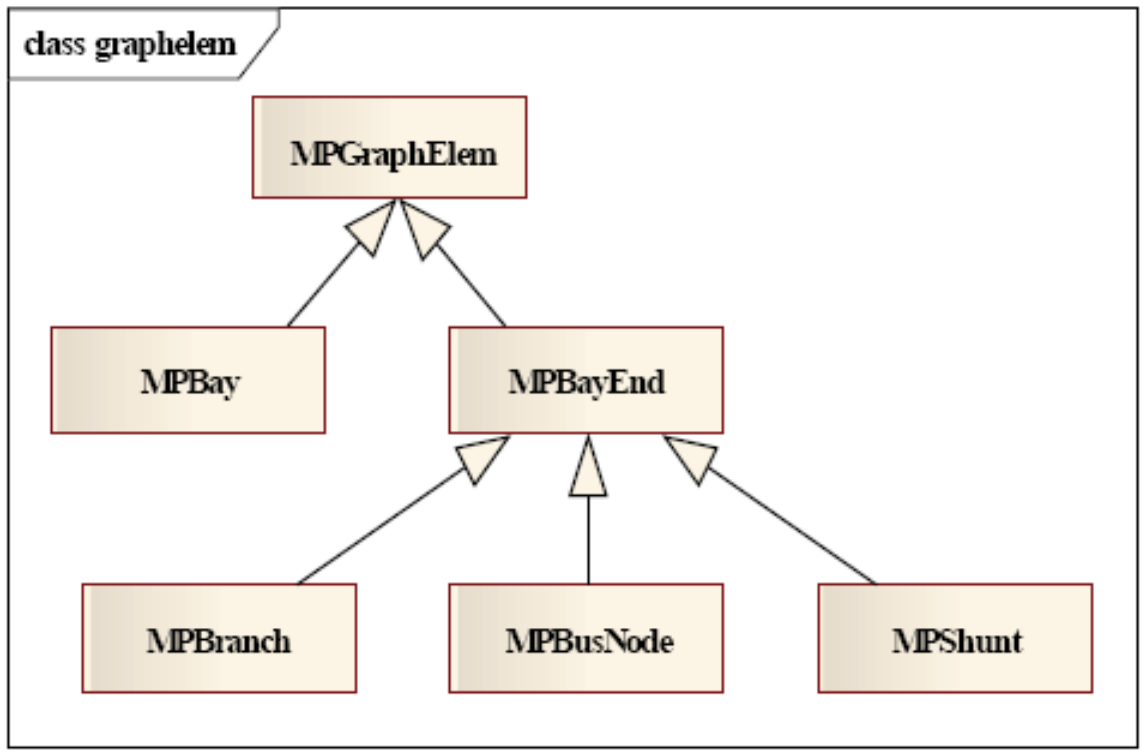

Figure 2 elementary classes of the internal model

There are clearly defined rules by which the elements of the graph are connected. They are given the following table. Each + sign indicates a permissible connection. [5-6]

Table 1 - Possible connections between elements MPGraphElem 
RADIAL STRUCTURE GRAPH -BASED MATHEMATICAL MODEL FOR LARGE DISTRIBUTION NETWORKS

\begin{tabular}{|c|c|c|c|c|}
\hline & MPBay & MPBusNode & MPBranch & MPShunt \\
\hline MPBay & & + & + & + \\
\hline MPBusNode & + & & + & + \\
\hline MPBranch & + & + & & \\
\hline MPShunt & + & + & & \\
\hline
\end{tabular}

\subsubsection{MPGraphElem}

Class MPGraphElem models elements of the graph of the distribution network. This class uniquely identifies each element modeled in the internal model. It also contains an attribute phases, which determines the phasing element and represents the structural information of each element. Elements of the graph form fields and elements that are connected across the field, the so-called end of the field.

\subsubsection{MPBayEnd}

Class MPBayEnd inherits class MPGraphElem. It represents the base class that models the ends of fields: nodes, branches and shunts. The class has an attribute phaseMarker that determines active phased elements, or those stages that are energized.

\subsubsection{MPBranch}

Class MPBranch inherits class MPBayEnd. This class models a branch graph DN. Branches are forked. The graph network, it is located between two nodes. The attribute endNode [2] refers to the objects of class MPBusNode. These are the nodes from the ends of the branches, which can be seen in the graph network. The first node of a series is always filled with a knot that is closer to the source, and is called the overhand knot.

A node branches which is farther from the origin is called the lower node, and it is to be completed by the second element of the array endNode. The most important class derived from the class MPBranch MPSection and MPTransformer. Class MPSection modeles the section, and the class MPTransformer two-winding and three-winding transformers.

\subsubsection{MPBusNode}

Class MPBusNode inherits class MPBayEnd and models the nodes of the graph. The attribute neighbors presenting the author vector elements graph incidents given node. The attribute parentChild presenting the author vector fields over the incident given node. It differs from the attribute neighbors, because it does not contain fields. This vector is filled during processing topologies and provides fast and efficient access to branches incident node that is observed.. Depending on whether the node branches further or closer to the root of the network, a branch of the parent or the child branch of a given node

\subsubsection{MPS hunt}

Class MPS Hunt inherits MP Beyond and modeled the following elements: a generator, motor and capacitor. These elements are collectively referred to as shunts. 
Book of Proceedings International Conference on Mechatronics, Sciences in Energy Efficiency Engineering, System Engineering and Robotics

\subsubsection{MPBay}

Class MPBay is derived from the class MPGraphElem. This class models the fields that connect the ends of the field. Each field has its own internal and external elements. The external elements are of the end of fields and represent objects of class MPBayEnd. Ends attribute contains information about the outside end of the field. The attribute equipment contains information about internal elements fields. Internal components are modeled class MPBayEquip. They make the equipment:

- switching devices,

- measuring transformers (voltage and current)

- fault detectors,

- relays,

- Surge venters.

\subsection{Navigate through the network}

Network Elements, on the basis of which creates an internal model, are placed in appropriate vectors. These vectors have the following characteristics:

- very quick access elements,

- ability to efficiently add / remove element,

- abilities copying elements.

For very fast access to these for efficient movement through the network, are defined classes and their methods. representing the so-called iterators. There are several different classes, suitable for processing network elements and pass through the same. These are:

- DownScanner

- ShuntScanner

- ScanIterator

- DownIterator

- UpIterator

- ContourIterator

- Alliterator.

Each of these classes has defined methods Begin and Next. The most important class of these groups is the class DownScanner. [7]

\subsubsection{DownScanner}

Class DownScanner carries a move through the network in a given direction, by Layer. This iterator leads over any other, carrying out preparation for updating the data elements given network, after which enables the use of other iterator. For this reason, it is much slower than the others, thus, is not used in energy functions. Reference is made in the processing of network topology for all roots detected islands. DownScanner while passing through a network it observers' vector neighbors for each node, while other iterators use vector parentChild. Before processing topology vector neighbors is only filled while the vector parentChild updated on the basis of the evaluation program. Due to this, iterators can be used after a call scanner, or after processing the topology.

\subsection{The topology analyzer}


RADIAL STRUCTURE GRAPH -BASED MATHEMATICAL MODEL FOR LARGE DISTRIBUTION NETWORKS

The topology analyzer, (processing topology) runes topological analysis of the current status using class DownScanner and internal model. Based on the status of switching equipment static elements of the network topology analyzer represents the distribution network in the form of a graph that represents the input for each energy function. Updating changes in the graph of the distribution network that are the consequences of changing the status of switching equipment or installation of a new element in this network, topology analyzer can be divided into two parts:

- processing topology by roots

- processing topology the islands.

The first deals with all the roots, and then detected the island.[7]

\section{CONCLUSION}

By presenting the distribution network in the form of a graph of the radial structure, which consists of nodes branches, enabled the implementation of specialized algorithms for compensating calculation of stationary regime and fault analysis. Advantages of these algorithms, as compared to other algorithms for the calculation of the regime in the distribution network, are much shorter time needed for calculating and a much higher accuracy of the calculation results. These benefits of compensating algorithms in particular are manifested when increasing the number of nodes that are part of the graph of the distribution network. Since the calculation of the function of the stationary regime and fault analysis form the basis for the work of all other energy functions, "Mathematical model" is a function that ensures compatibility integrity of the system energy applications

\section{Reference}

1. usa.siemens.com/smartgrid

2. https://www.capgemini.com/utilities/smart-energy-services

3. Piotr Grzybowski1, Cezary Mazurek1, Paweł Spychała 1, Marcin Wolski1 1, Data

Management System for grid and portal services Poznan Supercomputing and Networking

Center, ul. Noskowskiego 10, 61-704 Poznan, Poland \{piotrgrb, mazurek, spychala, maw\}@man.poznan.pl

4. Proposed IEC 61968 Standard Based Utility Architecture as referred from IEC Standard Documents, http://distributionmanagementsystem.blogspot.com/2011/06/iec-61968-standardsbased-dms.html

5. Asian Business Consortium, Engineering International, Volume 1, No 1 (2013)

6. Electricity North West/Respond/Configuration of NMS and installation of FLAT software V1.0/30 September 2016

7. https://www.cs.cmu.edu/ pattis/15-1XX/15-200/lectures/collectionsi/lecture.html 